\title{
Preliminary study of vibration characteristics of intelligent beam with the influence of SMA's restoring force
}

\author{
Chuanliang Shen ${ }^{1}$, Wei Su${ }^{1, a}$, Guochao Sun ${ }^{1}$, Binyu Zhou ${ }^{1}$, Tairong Sun ${ }^{1}$ and Yi Liu ${ }^{1}$ \\ ${ }^{1}$ State Key Laboratory of Automotive Simulation and Control, Jilin University, Changchun 130025, China
}

\begin{abstract}
In order to solve the problem of noise and comfort caused by vibration during automobile driving, this paper proposes to use shape memory alloy wire as driving element and simplified beam structure to form intelligent beam composite structure, and use the restoring force generated by shape memory alloy wire to realize vibration control of the structure. several macroscopic phenomenological constitutive models of NiTi alloy were summarized and analyzed. On the basis of the Liang-Rogers onedimensional constitutive model, Simulating the response force changing process of the SMA wire by useing MATLAB. According to the oretical analysis, we can obtain the system stiffness matrix and mass matrix, establish the dynamic model of smart beam composite structure, and analyze the interaction between shape memory composite wire prestress and natural frequency. The numerical simulation method is used to establish the simulation model and study the influence of the size and position parameters of the intelligent beam structure and the restoring force on its vibration characteristics. The results show that the natural frequency of the beam decreases and the natural frequency of the SMA wire increases with the increasing restoring force of SMA wire. As the distance from the SMA wire to the neutral axis increases, the natural frequency of the system increases, revealing the mechanism of vibration control of the shape memory alloy smart beam composite structure.
\end{abstract}

\section{Introduction}

Automobile vibration and noise are the important indexes that affect the ride comfort of the occupant. Serious car vibration and interior noise will also affect the safety of the vehicle. The automobile body is mainly composed of thin-walled panel and beam structure, which are connected to each other and constitute the overall frame structure of the automobile body. It is very necessary to restrain the vibration of body beam structure because the vibration of body beam will affect the ride comfort. The strength and stiffness of the structure may be adaptive if the shape memory alloy be used as the driving force of execution device compound or embedded into the structure. There are various deformation under the external excitation, elastic modulus can be changed along with the phase change, and great restoring force can be generated when limited recovery is achieved, so as to effectively suppress the vibration of the body structure.

When SMA plays the shape memory effect, the material can generate recovery stress of up to $700 \mathrm{MPa}$ and strain of about $8 \%{ }^{[1]}$. The huge recovery force generated when limited recovery is used can change the stiffness characteristics of the structure, make the structure's own frequency avoid the external excitation frequency, and reduce the vibration response of the structure. At the same time, the elastic modulus of SMA wire is increased by more than 3 times after the phase transformation under heat, which can increase the local or overall stiffness of structure ${ }^{[1,2]}$.In 1989, Rogers ${ }^{[3]}$ first made theoretical analysis of SMA applied in vibration

\footnotetext{
${ }^{\text {a }}$ Corresponding author: 3339177157@qq.com
}

control of composite structures. According to different modes of vibration suppression, SMA was used for structural vibration control into active control, passive control, semi-active control and mixed control ${ }^{[4]}$.Using the shape memory effect of SMA wire, McGavini ${ }^{[5]}$ applied it to the vibration suppression of frame structure and carried out experimental verification. The test results showed that the restoration force generated by SMA wire under heat can effectively change the natural frequency of frame structure, and the fundamental frequency of the driven system increased by $32 \%$.Jaakko Heinomen et al. explained the principle of SMA for semi-active control in a single-degree of freedom system in detail, activated SMA when the system frequency was $2.4 \mathrm{hz}$, and increased the stiffness property of the structure ${ }^{[6]}$. By stopping heating SMA at $7.5 \mathrm{hz}$, the stiffness of the structure is reduced, and the amplitude of the system is effectively reduced. Then, carried out the simulation of semi-active control of the intelligent system by using ABAQUS, and obtained the same damping effect. Barbara Tiseo et al. made an in-depth study on the change of stiffness caused by the change of the elastic modulus of SMA wire, and designed an ATDVA device ${ }^{[7]}$. The working mechanism was: when the SMA wire was heated, the NiTi alloy wire changed from martensite to austenite, the elastic modulus increased, and the stress inside the wire in the limited recovery increased. The structure is applied to the design of aviation industry, and aluminium alloy plate and glass fibre can effectively suppress vibration. In existing research for restoring force effect on the vibration of the beam structure study is infrequently.In existing research for restoring force effect 
on the vibration of the beam structure is infrequent.In this paper, the finite element model of the composite structure is established, this structure contains intelligent beam with shape memory alloy wire and simple support beam. The influence law between the restoring force of shape memory alloy wire and the vibration characteristics of intelligent beam is analyzed through numerical analysis and simulation, to reveal the mechanism of vibration control.

\section{Modeling of intelligent beam structure}

\subsection{Simplification of intelligent beam structure model}

The simplification of the shape memory alloy intelligent beam composite structure and the discretization of the structure can make it convenient to describe the engineering problem and use time variable to describe the mathematical model of the discrete system in the dynamic problem. The motion equation can also be expressed in the form of differential. In this paper, the beam -SMA structure system is discretized into a finite number of $n$ elements. The main mechanical behaviours of each element (such as strain, stress, displacement, etc.) can be expressed by the displacement of the nodes on the element. The mechanical behaviours of the system can be described by the displacement of all nodes. It is assumed that both ends of each unit are connected with prestressed SMA filaments, as shown in figureure 1.The length of the element is $L_{i}$, and the nodes at both ends are $\mathrm{i}$ and j. $V_{i}$ and $V_{j}, M_{i}$ and $M_{j}$ respectively represent the external force and torque applied on node $\mathrm{i}$ and $\mathrm{j}, \mathrm{M}_{\mathrm{N}}$ is the control torque generated by SMA wire.

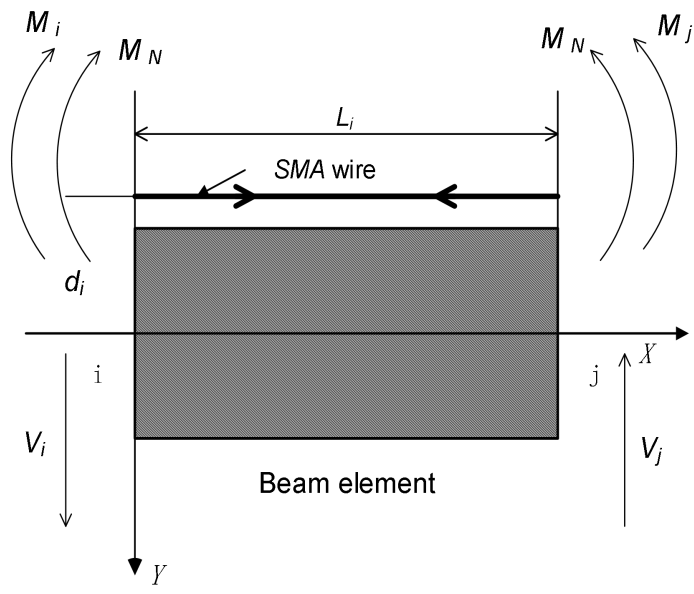

figure 1. beam -SMA unit diagram.

\subsection{Natural frequency of the system}

The dynamic equation of the beam -SMA system can be described as ${ }^{[8]}$ :

$$
M \ddot{\delta}+C \dot{\delta}+K \delta=F
$$

$\mathrm{M} 、 \mathrm{C} 、 \mathrm{~K}$ are the Mass matrix, damping matrix and stiffness matrix respectively. $\delta$ is the displacement vector of the whole system.

If the effect of damping and external load is ignored, the above equation of vibration differential equation of linear time-invariant system can be described as:

$$
\begin{gathered}
{[M]\{\ddot{\delta}\}+[K]\{\delta\}=0} \\
\{\delta\}=\{\varphi\}_{\mathrm{i}} \cos \omega_{i}
\end{gathered}
$$

Then

$$
\begin{gathered}
\left([K]-\omega_{i}{ }^{2}[M]\right)\{\varphi\}_{i}=\{0\} \\
\{\varphi\}_{i} \neq 0 \\
{[\mathrm{~K}]-\omega_{i}^{2}[M]=0} \\
f_{i}=\frac{\omega_{i}^{2}}{2 \pi} \quad(i=1,2,3 \cdots)
\end{gathered}
$$

In the above equation, $\omega_{i}$ is the natural circular frequency (radian/second) of the beam-SMA structure, $f_{i}$ is the natural frequency, and the mass matrix and stiffness matrix of the structure determine the natural frequency of the system. Considering that small deformation of the structure will not change the stiffness characteristics of the structure, the structure mass matrix $[\mathrm{M}]$ is not affected by the temperature, and the material properties and restoring force of the stiffness matrix $[\mathrm{K}]$ are all functions of the temperature. It can be seen that the variation of SMA wire temperature causes the change of structural stiffness and changes the vibration characteristics of the structure.

\section{Analytical analysis of intelligent beam structure}

\subsection{Relationship between pressure and frequency}

The restoring force generated by SMA wire can be regarded as the axial force exerted on both ends of the simple beam structure, regardless of whether the axial force is eccentric or not. Based on ignoring shear and rotational inertia, the bending vibration balance equation of the simple beam is as follows ${ }^{[9]}$ :

$$
\frac{\partial^{2}}{\partial x^{2}}\left(E I \frac{\partial^{2} y}{\partial x^{2}}\right)+N \frac{\partial^{2} y}{\partial x^{2}}+\bar{m} \frac{\partial^{2} y}{\partial t^{2}}=0
$$

$E I$ is the combined stiffness of simply supported steel beam and prestressed SMA wire. In other words, $E I=$ $E_{C} I_{C}+E_{P} I_{P}, N$ is the axial pressure generated by SMA wire, $\mathrm{m}$ is the quality of simply supported steel.

The solution is assumed to be: 


$$
y(x, t)=\varphi(x) q(t)
$$

$\varphi(x)$ is a function of $x, \mathrm{q}(\mathrm{t})$ is a function of $\mathrm{t}$.

Then:

$$
\frac{\mathrm{EI}^{\prime \prime \prime \prime}(x)}{\Phi(x)}+\frac{N \Phi^{\prime \prime}(x)}{\Phi(x)}=-\bar{m} \frac{\ddot{q}(t)}{q(t)}=\mu^{4}
$$

According to the above equation, the following two equations can be obtained:

$$
\begin{gathered}
\ddot{q}(t)+\omega^{2} q(t)=0 \\
I \Phi^{\prime \prime}(x)+N \Phi^{\prime \prime}(x)-\bar{m} \omega^{2} \Phi(x)=0
\end{gathered}
$$

It can be solved:

$$
\omega_{n}=\frac{n^{2} \pi^{2}}{l^{2}} \sqrt{\left(1-\frac{N I^{2}}{n^{2} \pi^{2} E I}\right)} \sqrt{\frac{E I}{m}}
$$

The above formula is the calculation formula of beam frequency when the beam is subjected to axial force. EI is the combined stiffness of SMA wire and beam. It can be seen from equation (13), the vibration frequency of the beam decreases with the increasing pre-stress.

\subsection{Relationship between SMA wire tension and frequency}

The tension force generated by the heat recovery of the SMA wire is applied to the beam, causing the whole structure of the beam to be compressed.For the whole structure of simple supported beams with prestress, the tension of restoring force on SMA itself should be considered at the same time. The restoring force is regarded as the axial tension on both ends of SMA wire, and its motion differential equation is as follows ${ }^{[10]}$ :

$$
\frac{N \partial^{2} y}{\partial x^{2}}+\rho_{P} A_{P} \frac{\partial^{2} y}{\partial t^{2}}=0
$$

$\rho_{P} A_{\mathrm{P}}$ is the mass of the pre-stressed SMA wire of unit length, this value is small relative to the steel beam structure. The calculation formula of pre-stressed SMA wire frequency under axial tension is as follows:

$$
\omega_{n}^{P^{2}}=\left(\frac{n \pi}{l}\right)^{2} \frac{N}{\rho_{P} A_{P}}
$$

According to the above equation, as the axial restoring force of SMA filament increases, its natural frequency increases.

\section{Numerical analysis and results}

\subsection{Influence of shape memory alloy wire regeneration force on the natural frequency of the system}

In this section, a numerical model of simple support beam and SMA wire is established by using MATLAB software, as shown in figureure 2.The size of the beam is $500 \mathrm{~mm} \times 50 \mathrm{~mm} \times 0.7 \mathrm{~mm}$, the diameter of SMA wire is $0.5 \mathrm{~mm}$ and the number of SMA wires is 3 , and the vertical distance $d$ of SMA wire to the neutral axis of the beam is $1.35 \mathrm{~mm}$.

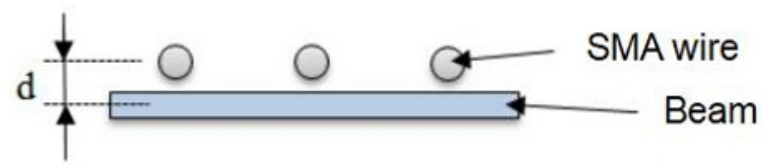

figure2. Cross section diagram of simple beam -SMA intelligent structure.

In the process of meshing, a lot of irregular units will be generated if you use a circular SMA wire. Leading the calculation time increased. In this paper, the length, arrangement and restoring force of SMA materials are considered as the main factors that affect the inhibition performance of SMA, ignoring the influence of section shape. Therefore, the square section is adopted to simulate the alloy wire, the section side length is $0.5 \mathrm{~mm}$, which shortens the calculation time and improves the calculation accuracy. Every initial strain of the shape memory alloy wire is $6.31 \%$, with the method of node coupling, bonding together five discrete coupling collection and the metal plate, create a contact between SMA wire and metal plate. The finite element model is shown as figure 3.

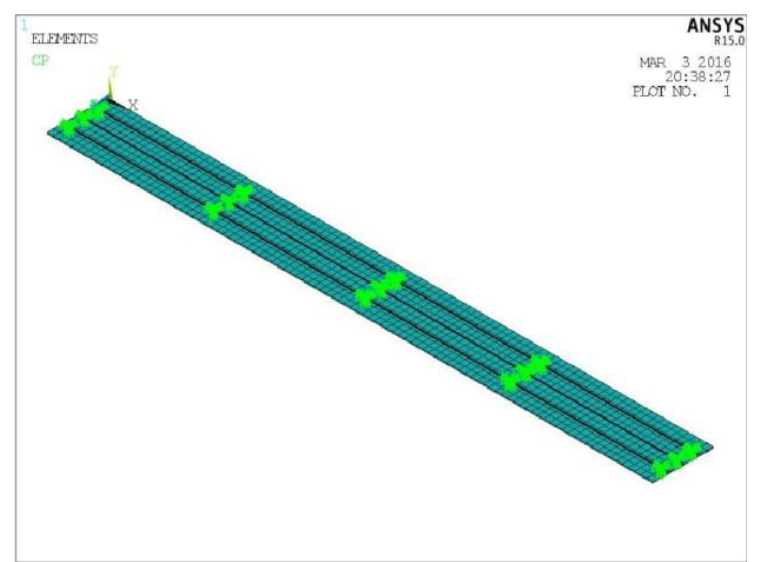

figure 3. Finite element model of both ends of fixed - supported beam with SMA wires arranged on the surface

In practice, each SMA wire is connected to the beam through 5 bolts and uniformly distributed, the arrangement is shown in the figure 4.

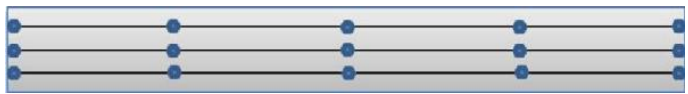

figure 4. Schematic diagram of connecting SMA wire and plate with bolts

As can be seen from figure 5, with the increasing restoring force of SMA wire, the first third order natural frequency of the beam decreases, and the first order frequency decreases obviously, and the natural frequency of SMA wire increases gradually, and the frequency of SMA wire increases significantly compared with beam. 


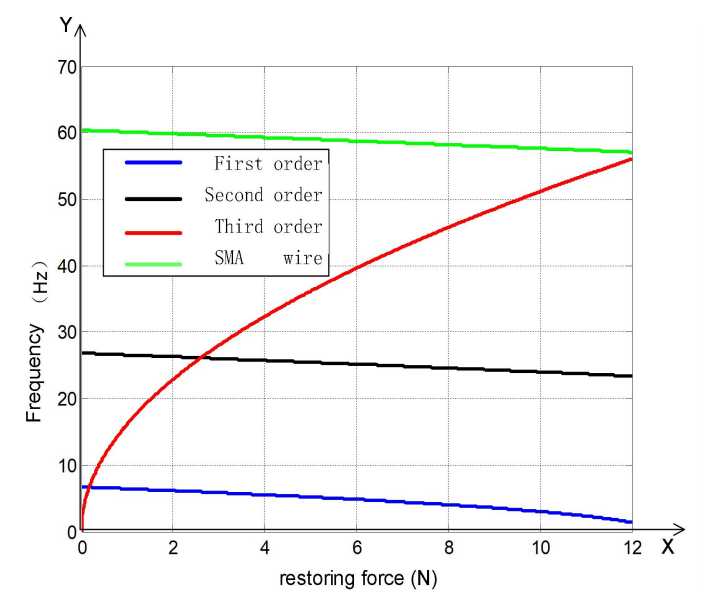

figure5. Relation of beam and SMA wire frequency with restoring force

\subsection{Influence of distance location parameters on the natural frequency of the system}

In order to study the effect of SMA wire placement on the natural vibration frequency of steel beam, numerical simulation was conducted for SMA wire at different .

As can be seen from figureure 6, as the distance from the SMA wire to the neutral axis of the beam increases, the natural frequency of the system increases significantly. Therefore, in order to enhance the SMA control effect on the variable stiffness of the body beam structure, it should be positioned away from the neutral layer of the beam. In the following simulation and experiment; the vertical distance from the SMA wire to the neutral axis of the beam is $2 \mathrm{~mm}$.eccentric position $\mathrm{d}$, and the analysis results are shown in figure 6.

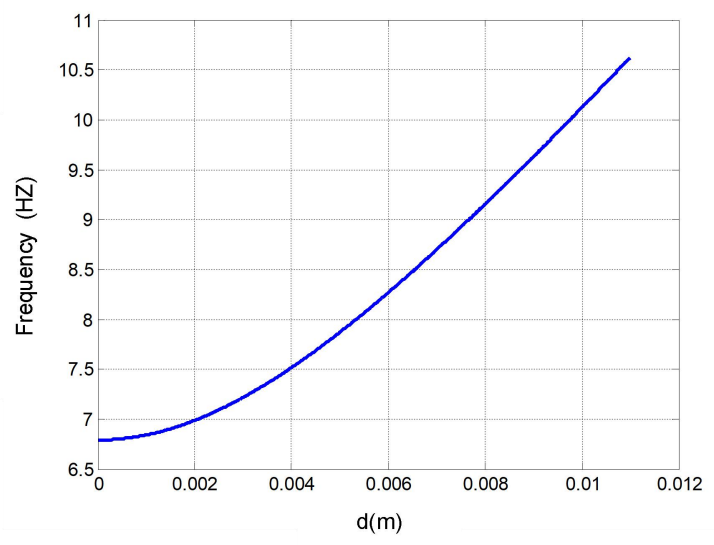

figure 6. Relation of system frequency with the neutral wheelbase distance from SMA wire to beam

\section{Conclusion}

The above content analyzed the relationship between the restoring force of SMA wire and the beam frequency theoretically, and the system dynamics model is established. Numerical simulation was carried out by using the numerical simulation software. the results showed that with the increasing restoring force of SMA wire, the first third order natural frequency of the beam decreased, and the first order frequency decreased significantly. As the distance from the SMA wire to the beam's neutral axis increases, the natural frequency of the system increases, and the SMA wire's control effect on the variable stiffness of the body beam structure

increases. In the application, SMA wire should be positioned as far as possible away from the beam's neutral layer. By analyzing the effect of the restoring force of shape memory alloy on structural vibration characteristics, the feasibility of using shape memory alloy wire to control the semi-active vibration of beam structure with variable stiffness was verified.

\section{Acknowledgments}

This project is supported by National Natural Science Foundation of China (Grant No. 51875237).The authors are grateful for the financial support from National Natural Science Foundation of China (Grant No. 51875237), the Science and Technology Development Project of Jilin Province (Project No. 20160101283JC) and the "13th Five-Year" Science and Technology Research Project of Education Department of Jilin Province (Project No. 2016-417).

\section{References}

1. S.Gao,YP.Shen,D.G.Xu.Shape memory alloy and its mechanical analysis in engineering applications[FJ].Mechanical progress,3:301-312(1997)

2. T. Kobori, M. Takahashi, T. Nasu, Seismic response controlled structure with Active Variable Stiffness system[J]. Earthquake Engineering\&Structural Dynamics, 22(11):925-941(1993)

3.C. Rogers. Behavior of shape memory alloy reinforced composite plates. I - Modelformulations and control concepts $[\mathrm{C}] / /$ 30th Structures. Structural Dynamics andMaterials Conference (1989)

4. H.Li, Application of shape memory alloy in structural vibration control[D 3 . Beijing jiaotong university (2007)

5. G. McGavin, G.Guerin. Real-time seismic damping and frequency structures using nitinol wire[C]. Proceedings of SPIE,4696:175-184(2002)

6. J. Heinonen, I. Vessonen, P. Klinge. Controlling stiffness of a frame spring by changing the boundary condition with an SMA actuator[J]. Computers\&Structures,86:398-406(2008)

7. B. Tiseo, A. Concilio, S. Ameduri. A shape memory alloys based tuneable dynamic vibration absorber for vibration tonal control[J]. Journal of Theoretical\&Applied Mechanics,48:135-153(2010) 8. M. Paz, H. Saunders. Structural Dynamics Theory and Computations[J].Journal of Vibration\&Acoustics, 107(4):319-347.(1985)

9. Y.G.Tang,Advanced structural mechanics[M].Tianjin university press(2002)

10.X.Yang,J.Hong,Y.H.Ma.Experimental study on vibration control of SMA smart beam 